\title{
ATP7B Mutation Analysis: Wilson Disease, A Difficult to Diagnose Case
}

\author{
Muhammad Almas Hashmi ${ }^{1}$, Bibi Zubaida ${ }^{2}$, Rai Muhammad Asghar ${ }^{3}$ and Munir Akmal Lodhi ${ }^{1}$ \\ ${ }^{1}$ Department of Pediatrics, Foundation University, Islamabad, Pakistan \\ ${ }^{2}$ Medical Genetics Research Laboratory, Department of Biotechnology, Quaid-i-Azam University, Islamabad, Pakistan \\ ${ }^{3}$ Department of Pediatrics, Rawalpindi Medical University and Allied Hospitals, Rawalpindi, Pakistan
}

\begin{abstract}
Wilson's Disease (WD) is a common metabolic disorder predominantly involving liver, brain, and eyes. Pancreatic, renal, psychiatric, and cardiac involvement have also been described. No single investigation can be considered diagnostic of WD; therefore, diagnosis is based upon a series of tests best interpreted using Wilson disease diagnostic index (WDDI). We present a difficult-to-diagnose, 9year girl of consanguineous parents, with chronic liver disease and portal hypertension. Initial workup was equivocal with significantly low serum ceruloplasmin, normal urinary copper excretion and absent Kaiyser-Fleischer (KF) rings. Diagnosis was established by ATP7B mutation analysis. The patient was found homozygous for C.3955C>T (p.Arg1319Ter) in exon 19, a rare mutation described in literature, which results in premature truncation of peptide chain.
\end{abstract}

Key Words: ATP7B, Wilson disease, Copper, Mutations, Hepatolenticular degeneration.

How to cite this article: Hashmi MA, Zubaida B, Asghar RM, Lodhi MA. ATP7B Mutation Analysis: Wilson Disease, A Difficult to Diagnose Case. J Coll Physicians Surg Pak 2020; 30(04):433-434. DOI: https://doi.org/10.29271/jcpsp.2020.04.433.

\section{INTRODUCTION}

Wilson's Disease (WD) is among the commonest genetic metabolic disorders of the liver. The gene responsible is ATP7B on chromosome $13 .{ }^{1}$ The gene product is an enzyme-essential in incorporation of copper into ceruloplasmin within the hepatocyte membrane and excretion in bile and outside the body through feces. ATP7B gene mutation results in buildup of copper in the body. ${ }^{2}$ The accumulated copper is deposited in the liver, cornea, brain and pancreas, resulting in protean manifestations ranging from liverdisease, central nervous system (CNS) disease, psychiatric manifestations, hemolysis, pancreatitis and cardiac manifestations. Most common presentation is with hepatic and neurological involvement. ${ }^{3}$

The diagnosis of WD is straight forward, based on serum ceruloplasmin, urinary copper excretion, hepatic copper content, and eye examination for Kaiyser-Fleischer (KF) rings in most cases. ${ }^{4}$ Homozygous mutation of ATP7B gene is considered diagnostic for WD. More than 500 disease causing mutations have been described in the literature. In doubtful cases, where biochemical and histopathological features are equivocal, mutation analysis can be extremely useful investigation for accurate diagnosis of WD.

We present a case ofWD with equivocal findings on routine tests in which the diagnosis was confirmed by mutation analysis.

Correspondence to: Dr. Muhammad Almas Hashmi, Department of Pediatrics, Foundation University, Islamabad, Pakistan

E-mail: almashashmi@hotmail.com

Received: May 25, 2019; Revised: August 16, 2019;

Accepted: August 26, 2019

DOI: https://doi.org/10.29271/jcpsp.2020.04.433

\section{CASE REPORT}

A 9-year female presented to the Paediatrics Department of Holy Family Hospital, Rawalpindi, with history of abdominal distension and jaundice for 3 months and altered conscious level for 2 days. She did not have fever, joint pains or skin rash. Her parents were first cousins and she had two elder female siblings, one younger female sibling and one younger male sibling. Rest of the siblings were alive and healthy. Child was in grade 2 hepatic encephalopathy at presentation, jaundiced having hepatosplenomegaly and ascites. Patient had normal blood counts. Serum bilirubin was $5.9 \mathrm{mg} / \mathrm{dL}$ (direct $2.6 \mathrm{mg} / \mathrm{dL}$ ). alanine aminotransferase (ALT) was $32 \mathrm{iu} / \mathrm{mL}$, aspartate aminotransferase (AST) $21 \mathrm{iu} / \mathrm{ml}$, gamaglutamyl transpeptidase ( $\mathrm{\gamma}-\mathrm{GT}$ ) $64 \mathrm{iu} / \mathrm{mL}$, serum albumin $2.5 \mathrm{mg} / \mathrm{dL}$ and normal coagulation profile (INR 1.1). Serum ceruloplasmin was $7.7 \mathrm{mg} / \mathrm{dL}$ (range: $20-35 \mathrm{mg} / \mathrm{dL}$ ), which was in favour of WD but urinary copper with penicillamine challenge was $140 \mathrm{ug} / 24$ hours, which was only mildly raised (normal ug $<50$ ug/24 hours) and $\mathrm{KF}$ rings were absent. Workup for autoimmune hepatitis was unremarkable.

WD genetic mutation analysis was done at the Medical Genetics Research Laboratory, Department of Biotechnology, Quaid-iAzam University, Islamabad, Pakistan. Sanger sequencing of ATP7B gene identified a homozygous nonsense mutation c.3955C>T (p.Arg1319Ter) in exon 19 (Figure 1).

Patient improved with penicillaminc, zinc and supportive care and liver functions normalised over a period of 8 months.

\section{DISCUSSION}

WD is among the commonest causes of childhood liver diseases; and even fewer treatable causes. Diagnosis of WD is usually straight forward, on the basis of serum ceruloplasmin levels, 24hour urinary copper excretion estimation and the presence of $\mathrm{KF}$ 
rings on slit-lamp examination. Liver biopsy and calculation of copper content of dry weight of liver is not widely available. However, the diagnosis of WD is complicated because of subtle and diverse clinical features, and lack of accurate biochemical markers. ${ }^{5}$ Ceruloplasmin is an acute phase reactant and may be falsely negative in acute inflammation as well as in $15-36 \%$ of patients with WD and false positive in heterozygous individuals and aceruloplasminemia. KF rings are usually absent under 10 years of age. Copper estimation of dry weight of liver is an invasive test and not freely available. Urinary copper excretion is variable among individual patients.

Figure 1: Region of exon 19 of ATP7B gene from control individual (A). Region of exon 19 in proband (B). Arrows indicate the position of mutation.

Under these circumstances, mutation analysis becomes an extremely useful adjunct to the diagnostic testing. However, the large number of known mutations (>500) and a very large gene complicate the situation.

In a recent meta-analysis, p.His1069GIn was most often encountered mutation in Europe, while p.Arg788Leu was common in Far Feast. P.GIn1399Arg was commonest in Saudi Arabia, p.His1069GIn was dominant in USA, and c.3402 delC was predominant in Brazil. ${ }^{6}$ In another review, common mutations identified in North American, Russian and Swedish populations were single nucleotide substitution, c.3207C>A (His1069Gln) involving exon 14. Other slightly less common mutations were 3402 delC and c.3809A>G.(Asn1270Ser). ${ }^{7}$

The mutation identified in our index case, i.e. c.3955C>T (p.Arg1319Ter), was a single nucleotide substitution resulting in premature truncation of the peptide chain at amino acid 1319. The mutation has been reported as pathogenic and is considered as a rare mutation with only 10 patients registered in The University of Alberta Wilson's Disease mutation registry. The reported cases are mainly from England and European countries with one each from China and Egypt. No case has been reported from the IndoPaksubcontinent yet. ${ }^{8-10}$

It is inferred that mutation analysis is a useful adjunct to the diagnosis of WD because of wide clinical spectrum, subtle features and lack of accurate biochemical diagnostic tests. ATP7B gene is a very large gene, therefore, identification of common mutations in
Pakistani population will greatly help in genetic diagnosis ofWD.

PATIENTS' CONSENT:

Informed Consent was taken from the father of the patient.

\section{CONFLICT OF INTEREST:}

Authors declared no conflict of interest.

\section{AUTHORS' CONTRIBUTION:}

$\mathrm{MAH}$ : Conceptualization, data collection, write-up. BZ: Genetic testing, literature review.

RMA, MAL: Critical review, proofreading.

\section{REFERENCES}

1. Bandmann O, Weiss KH, Kaler SG. Wilson's disease and other neurological copper disorders. Lancet Neurol 2015; 14: 103-13.

2. Ferenci P. Diagnosis of wilson disease. Handb Clin Neurol 2017; 142:171-80.

3. Schilsky ML. Wilson disease diagnosis, treatment and followup. Clin Liver Dis 2017; 21:755-67.

4. Ferenci P, Caca K, Loudianos G, Mieli-Vergani G, Tanner S, Sternlieb I, et al. Diagnosis and phenotypic classification of wilson disease. Liver Int 2003; 23:139-42.

5. European Association for Study of Liver. EASL clinical practice guidelines wilson's disease. J Hepatol 2012; 56:671-85.

6. Gomes A, Dedoussis GV. Geographic distribution of atp7b mutations in wilson disease. Ann Hum Biol 2016; 43:1-8

7. Ariöz $C$, Li Y, Wittung-Stafshede $P$. The six metal binding domains in human copper transporter atp7b molecular biophysics and disease-causing mutations. Biometals 2017; 30:823-40.

8. Figus A, Angius A, Loudianos G, Bertini C, Dessi V, Loi A, et. al. Molecular pathology and haplotype analysis of wilson disease in Mediterranean populations. Am J Hum Genet 1995; 57:1318-24.

9. Mak CM, Lam CW, Tam S, Lai CL, Chan LY, Fan ST, et al. Mutational analysis of 65 wilson disease patients in hong kong Chinese identification of 17 novel mutations and its genetic heterogeneity. J Hum Genet 2008; 53:55-63.

10. Abdelghaffar TY, Elsayed SM, Elsobky E, Bochow B, Büttner J, Schmidt $\mathrm{H}$. Mutational analysis of atp $7 \mathrm{~b}$ gene in egyptian children with wilson disease 12 novel mutations. J Hum Genet 2008; 53:681-7. 